\title{
Global well-posedness of 2D generalized MHD equations with fractional diffusion
}

Zhiqiang Wei ${ }^{1 *}$ and Weiyi Zhu

${ }^{*}$ Correspondence:

wei.zhiqiang@yahoo.com

1 School of Mathematics and Information Sciences, North China University of Water Resources and Electric Power, Zhengzhou, 450011, P.R. China

Full list of author information is available at the end of the article

\section{Abstract}

In this paper we prove the uniqueness of weak solutions and the global-in-time existence of smooth solutions of the 2D generalized MHD system with fractional diffusion with $\frac{1}{2}$ power.

MSC: 35Q30; 76D03; 76D09

Keywords: MHD; fractional diffusion; uniqueness; smooth solution

\section{Introduction}

In this paper, we consider the following 2D generalized MHD system with $0<\alpha \leq 1$ [1]:

$$
\begin{aligned}
& \operatorname{div} u=\operatorname{div} b=0, \\
& \partial_{t} u+(u \cdot \nabla) u+\nabla\left(\pi+\frac{1}{2}|b|^{2}\right)+(-\Delta)^{\alpha} u=b \cdot \nabla b, \\
& \partial_{t} b+u \cdot \nabla b-b \cdot \nabla u-\Delta b=0, \\
& (u, b)(t=0)=\left(u_{0}, b_{0}\right) .
\end{aligned}
$$

Here, $u$ is the fluid velocity field, $\pi$ is the pressure and $b$ is the magnetic field.

Very recently, Ji [1] used the Fourier series analysis motivated in [2] to prove the global-in-time existence of smooth solutions of problem (1.1)-(1.4) when $\frac{1}{2}<\alpha \leq 1$, and Ji [1] pointed out that his result did not seem to come directly from the method like energy estimates. In this paper, we use the standard energy method to deal with the case $\alpha=\frac{1}{2}$; of course, our method also works when $\alpha>\frac{1}{2}$. We will prove the following.

Theorem 1.1 Let $\alpha=\frac{1}{2}$. Let $u_{0}, b_{0} \in H^{1}$ with $\operatorname{div} u_{0}=\operatorname{div} b_{0}=0$ in $\mathbb{R}^{2}$. Then problem (1.1)(1.4) has a unique global-in-time weak solution $(u, b)$ satisfying

$$
(u, b) \in L^{\infty}\left(0, T ; H^{1}\right), \quad u \in L^{2}\left(0, T ; H^{3 / 2}\right), \quad b \in L^{2}\left(0, T ; H^{2}\right)
$$

for any $T>0$.

Theorem 1.2 Let $\alpha=\frac{1}{2}$. Let $u_{0}, b_{0} \in H^{s}$ with $s>1$ and $\operatorname{div} u_{0}=\operatorname{div} b_{0}=0$ in $\mathbb{R}^{2}$. Then problem (1.1)-(1.4) has a unique global-in-time smooth solution $(u, b)$ satisfy-

\section{囪 Springer}

(c2013 Wei and Zhu; licensee Springer. This is an Open Access article distributed under the terms of the Creative Commons Attribution License (http://creativecommons.org/licenses/by/2.0), which permits unrestricted use, distribution, and reproduction in any medium, provided the original work is properly cited. 
ing

$$
u, b \in L^{\infty}\left(0, T ; H^{s}\right), \quad u \in L^{2}\left(0, T ; H^{s+\frac{1}{2}}\right), \quad b \in L^{2}\left(0, T ; H^{s+1}\right)
$$

for any $T>0$.

For $3 \mathrm{D}$ case and other related problems, we refer to $[3,4]$.

Our proof will use the following commutator estimates due to Kato and Ponce [5]:

$$
\left\|\Lambda^{s}(f g)-f \Lambda^{s} g\right\|_{L^{p}} \leq C\left(\|\nabla f\|_{L^{p_{1}}}\left\|\Lambda^{s-1} g\right\|_{L^{q_{1}}}+\left\|\Lambda^{s} f\right\|_{L^{p_{2}}}\|g\|_{L^{q_{2}}}\right),
$$

with $s \geq 1, \Lambda:=(-\Delta)^{1 / 2}$ and $\frac{1}{p}=\frac{1}{p_{1}}+\frac{1}{q_{1}}=\frac{1}{p_{2}}+\frac{1}{q_{2}}$.

\section{Proof of Theorem 1.1}

This section is devoted to the proof of Theorem 1.1. The global-in-time existence of weak solutions satisfying $(1.5)$ was proved in $[1,6]$, we only need to show the uniqueness. Let $\left(u_{i}, \pi_{i}, b_{i}\right)(i=1,2)$ be two weak solutions of problem (1.1)-(1.4). We define

$$
\delta u:=u_{1}-u_{2}, \quad \delta \pi:=\pi_{1}-\pi_{2}, \quad \delta b:=b_{1}-b_{2} .
$$

Then it follows from (1.1)-(1.3) that

$$
\begin{aligned}
& \operatorname{div} \delta u=0, \quad \operatorname{div} \delta b=0, \\
& \partial_{t} \delta u+u_{1} \cdot \nabla \delta u+\delta u \cdot \nabla u_{2}+\nabla\left(\pi+\frac{1}{2}\left(b_{1}^{2}-b_{2}^{2}\right)\right)+(-\Delta)^{1 / 2} \delta u \\
& \quad=b_{1} \cdot \nabla \delta b+\delta b \cdot \nabla b_{2}, \\
& \partial_{t} \delta b+u_{1} \cdot \nabla \delta b+\delta u \cdot \nabla b_{2}-b_{1} \cdot \nabla \delta u-\delta b \cdot \nabla u_{2}-\Delta \delta b=0 .
\end{aligned}
$$

Testing (2.2) by $\delta u$ and using (1.1) and (2.1), we see that

$$
\begin{aligned}
\frac{1}{2} \frac{d}{d t} \int|\delta u|^{2} d x+\int\left|\Lambda^{1 / 2} \delta u\right|^{2} d x= & -\int \delta u \cdot \nabla u_{2} \cdot \delta u d x \\
& +\int b_{1} \cdot \nabla \delta b \cdot \delta u d x+\int \delta b \cdot \nabla b_{2} \cdot \delta u d x \\
=: & I_{1}+I_{2}+I_{3} .
\end{aligned}
$$

Testing (2.3) by $\delta b$ and using (1.1) and (2.1), we find that

$$
\begin{aligned}
\frac{1}{2} \frac{d}{d t} \int|\delta b|^{2} d x+\int|\nabla \delta b|^{2} d x= & -\int \delta u \cdot \nabla b_{2} \cdot \delta b d x \\
& +\int b_{1} \cdot \nabla \delta u \cdot \delta b d x+\int \delta b \cdot \nabla u_{2} \cdot \delta b d x \\
=: & I_{4}+I_{5}+I_{6} .
\end{aligned}
$$


In the following calculations, we use the Sobolev embedding $\dot{H}^{1 / 2} \subset L^{4}$ and the Gagliardo-Nirenberg inequalities

$$
\begin{aligned}
& \|w\|_{L^{8 / 3}}^{2} \leq C\|w\|_{L^{2}}\left\|\Lambda^{1 / 2} w\right\|_{L^{2}}, \\
& \|w\|_{L^{4}}^{2} \leq C\|w\|_{L^{2}}\|\nabla w\|_{L^{2}} .
\end{aligned}
$$

Using (1.1), (2.1), (1.5), (2.6) and (2.7), we bound $I_{1}, I_{2}+I_{5}, I_{3}+I_{4}$ and $I_{6}$ as follows:

$$
\begin{aligned}
& I_{1} \leq\left\|\nabla u_{2}\right\|_{L^{4}}\|\delta u\|_{L^{8 / 3}}^{2} \leq C\left\|u_{2}\right\|_{H^{3 / 2}}\|\delta u\|_{L^{2}}\left\|\Lambda^{1 / 2} \delta u\right\|_{L^{2}} \\
& \leq \frac{1}{16}\left\|\Lambda^{1 / 2} \delta u\right\|_{L^{2}}^{2}+C\left\|u_{2}\right\|_{H^{3 / 2}}^{2}\|\delta u\|_{L^{2}}^{2}, \\
& I_{2}+I_{5}=0 \\
& I_{3}+I_{4} \leq C\left\|\nabla b_{2}\right\|_{L^{4}}\|\delta u\|_{L^{2}}\|\delta b\|_{L^{4}} \\
& \quad \leq C\left\|\nabla b_{2}\right\|_{L^{4}}\|\delta u\|_{L^{2}}\|\delta b\|_{L^{2}}^{1 / 2}\|\nabla \delta b\|_{L^{2}}^{1 / 2} \\
& \quad \leq \frac{1}{16}\|\nabla \delta b\|_{L^{2}}^{2}+C\|\delta u\|_{L^{2}}^{2}+C\left\|\nabla b_{2}\right\|_{L^{4}}^{2}\|\delta b\|_{L^{2}}^{2}, \\
& I_{6} \leq\left\|\nabla u_{2}\right\|_{L^{2}}\|\delta b\|_{L^{4}}^{2} \leq C\|\delta b\|_{L^{4}}^{2} \leq C\|\delta b\|_{L^{2}}\|\nabla \delta b\|_{L^{2}} \\
& \leq \frac{1}{16}\|\nabla \delta b\|_{L^{2}}^{2}+C\|\delta b\|_{L^{2}}^{2} .
\end{aligned}
$$

Adding up (2.4) and (2.5) and using the above estimates, we conclude that

$$
\begin{aligned}
& \frac{1}{2} \frac{d}{d t} \int\left(|\delta u|^{2}+|\delta b|^{2}\right) d x \\
& \quad \leq C\left\|u_{2}\right\|_{\dot{H}^{3 / 2}}^{2}\|\delta u\|_{L^{2}}^{2}+C\|\delta u\|_{L^{2}}^{2}+C\left\|\nabla b_{2}\right\|_{L^{4}}^{2}\|\delta b\|_{L^{2}}^{2}+C\|\delta b\|_{L^{2}}^{2}
\end{aligned}
$$

which gives

$$
\delta u=\delta b=0 .
$$

This completes the proof.

\section{Proof of Theorem 1.2}

This section is devoted to the proof of Theorem 1.2. We only need to prove a priori estimates (1.6) for simplicity.

First, we have (1.5).

Applying $\Lambda^{s}$ to (1.2), testing by $\Lambda^{s} u$ and using (1.1), we see that

$$
\begin{aligned}
\frac{1}{2} \frac{d}{d t} \int\left|\Lambda^{s} u\right|^{2} d x+\int\left|\Lambda^{s+\frac{1}{2}} u\right|^{2} d x \\
=-\int\left(\Lambda^{s}(u \cdot \nabla u)-u \nabla \Lambda^{s} u\right) \Lambda^{s} u d x \\
\quad+\int\left(\Lambda^{s}(b \cdot \nabla b)-b \cdot \nabla \Lambda^{s} b\right) \Lambda^{s} u d x+\int b \cdot \nabla \Lambda^{s} b \cdot \Lambda^{s} u d x \\
=: J_{1}+J_{2}+J_{3} .
\end{aligned}
$$


Applying $\Lambda^{s}$ to (1.3), testing by $\Lambda^{s} b$ and using (1.1), we find that

$$
\begin{aligned}
\frac{1}{2} \frac{d}{d t} \int\left|\Lambda^{s} b\right|^{2} d x+\int\left|\Lambda^{s+1} b\right|^{2} d x \\
=-\int\left(\Lambda^{s}(u \cdot \nabla b)-u \cdot \nabla \Lambda^{s} b\right) \Lambda^{s} b d x \\
\quad+\int\left(\Lambda^{s}(b \cdot \nabla u)-b \cdot \nabla \Lambda^{s} u\right) \Lambda^{s} b d x+\int b \cdot \nabla \Lambda^{s} u \cdot \Lambda^{s} b d x \\
=: J_{4}+J_{5}+J_{6} .
\end{aligned}
$$

Using (1.7), (2.6), (2.7) and (1.5), we bound $J_{1}, J_{2}, J_{3}+J_{6}, J_{4}$ and $J_{5}$ as follows:

$$
\begin{aligned}
& J_{1} \leq C\|\nabla u\|_{L^{4}}\left\|\Lambda^{s} u\right\|_{L^{8 / 3}}^{2} \\
& \leq C\|u\|_{H^{3 / 2}}\left\|\Lambda^{s} u\right\|_{L^{2}}\left\|\Lambda^{s+\frac{1}{2}} u\right\|_{L^{2}} \\
& \leq \frac{1}{8}\left\|\Lambda^{s+\frac{1}{2}} u\right\|_{L^{2}}^{2}+C\|u\|_{\dot{H}^{3 / 2}}^{2}\left\|\Lambda^{s} u\right\|_{L^{2}}^{2} \\
& J_{2} \leq C\|\nabla b\|_{L^{4}}\left\|\Lambda^{s} b\right\|_{L^{2}}\left\|\Lambda^{s} u\right\|_{L^{4}} \\
& \leq C\|\nabla b\|_{L^{4}}\left\|\Lambda^{s} b\right\|_{L^{2}}\left\|\Lambda^{s+\frac{1}{2}} u\right\|_{L^{2}} \\
& \leq \frac{1}{8}\left\|\Lambda^{s+\frac{1}{2}} u\right\|_{L^{2}}^{2}+C\|\nabla b\|_{L^{4}}^{2}\left\|\Lambda^{s} b\right\|_{L^{2}}^{2}, \\
& J_{3}+J_{6}=0, \\
& J_{4}, J_{5} \leq C\|\nabla u\|_{L^{2}}\left\|\Lambda^{s} b\right\|_{L^{4}}^{2}+C\|\nabla b\|_{L^{8 / 3}}\left\|\Lambda^{s} u\right\|_{L^{8 / 3}}\left\|\Lambda^{s} b\right\|_{L^{4}} \\
& \quad \leq C\left\|\Lambda^{s} b\right\|_{L^{4}}^{2}+C\|\nabla b\|_{L^{8 / 3}}^{2}\left\|\Lambda^{s} u\right\|_{L^{8 / 3}}^{2} \\
& \quad \leq C\left\|\Lambda^{s} b\right\|_{L^{2}}\left\|\Lambda^{s+1} b\right\|_{L^{2}}+C\|\nabla b\|_{L^{8 / 3}}^{2}\left\|\Lambda^{s} u\right\|_{L^{2}}\left\|\Lambda^{s+\frac{1}{2}} u\right\|_{L^{2}} \\
& \quad \leq \frac{1}{8}\left\|\Lambda^{s+1} b\right\|_{L^{2}}^{2}+\frac{1}{8}\left\|\Lambda^{s+\frac{1}{2}} u\right\|_{L^{2}}^{2}+C\left\|\Lambda^{s} b\right\|_{L^{2}}^{2}+C\|\nabla b\|_{L^{8 / 3}}^{4}\left\|\Lambda^{s} u\right\|_{L^{2}}^{2} .
\end{aligned}
$$

Adding up (3.1) and (3.2) and using the above estimates, we arrive at

$$
\begin{aligned}
& \frac{d}{d t} \int\left(\left|\Lambda^{s} u\right|^{2}+\left|\Lambda^{s} b\right|^{2}\right) d x+\int\left(\left|\Lambda^{s+\frac{1}{2}} u\right|^{2}+\left|\Lambda^{s+1} b\right|^{2}\right) d x \\
& \quad \leq C\|u\|_{\dot{H}^{3 / 2}}^{2}\left\|\Lambda^{s} u\right\|_{L^{2}}^{2}+C\|\nabla b\|_{L^{4}}^{2}\left\|\Lambda^{s} b\right\|_{L^{2}}^{2}+C\left\|\Lambda^{s} b\right\|_{L^{2}}^{2}+C\|\nabla b\|_{L^{8 / 3}}^{4}\left\|\Lambda^{s} u\right\|_{L^{2}}^{2},
\end{aligned}
$$

which yields (1.6).

This completes the proof.

Competing interests

The authors declare that they have no competing interests.

\section{Authors' contributions}

ZW proposed the problems and finished the whole manuscript. WZ modified the proofs. All authors read and approved the final manuscript.

\section{Author details}

'School of Mathematics and Information Sciences, North China University of Water Resources and Electric Power, Zhengzhou, 450011, P.R. China. ${ }^{2}$ Department of Mathematics, Zhejiang Normal University, Jinhua, Zhejiang 321004 P.R. China. 
Received: 9 August 2013 Accepted: 30 August 2013 Published: 07 Nov 2013

\section{References}

1. Ji, E: On two-dimensional magnetohydrodynamic equations with fractional diffusion. Nonlinear Anal. 80, 55-65 (2013)

2. Mattingly, JC, Sinai, YG: An elementary proof of the existence and uniqueness theorem for the Navier-Stokes equations. Commun. Contemp. Math. 1, 497-516 (1999)

3. Zhou, Y: Regularity criteria for the generalized viscous MHD equations. Ann. Inst. Henri Poincaré, Anal. Non Linéaire 24(3), 491-505 (2007)

4. Zhou, Y, Fan, J: A regularity criterion for the $2 \mathrm{D}$ MHD system with zero magnetic diffusivity. J. Math. Anal. Appl. 378(1), 169-172 (2011)

5. Kato, T, Ponce, G: Commutator estimates and the Euler and Navier-Stokes equations. Commun. Pure Appl. Math. 41, 891-907 (1988)

6. Cao, C, Wu, J: Global regularity for the $2 \mathrm{D}$ MHD equations with mixed partial dissipation and magneto diffusion. Adv. Math. 226, 1803-1822 (2011)

10.1186/1029-242X-2013-489

Cite this article as: Wei and Zhu: Global well-posedness of 2D generalized MHD equations with fractional diffusion. Journal of Inequalities and Applications 2013, 2013:489

Submit your manuscript to a SpringerOpen ${ }^{\circ}$ journal and benefit from:

- Convenient online submission

- Rigorous peer review

- Immediate publication on acceptance

- Open access: articles freely available online

- High visibility within the field

- Retaining the copyright to your article

Submit your next manuscript at $>$ springeropen.com 